
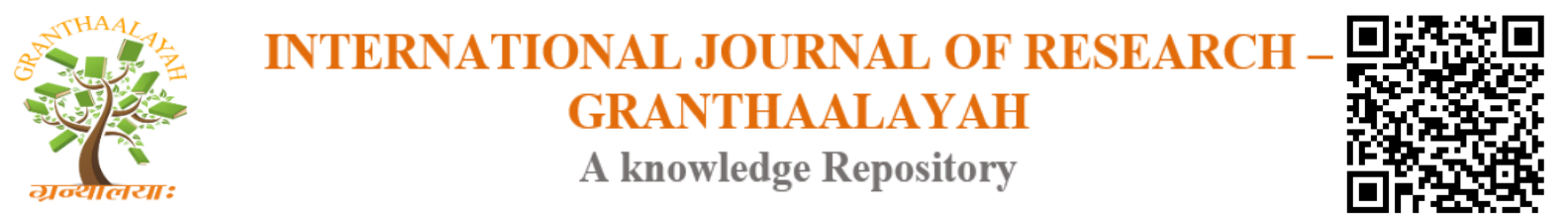

Science

\title{
ANTIMICROBIAL RESISTANCE: REVIEW
}

\author{
Daba Gudata ${ }^{1}$, Feyissa Begna ${ }^{2}$ \\ ${ }^{1,2}$ Jimma University College of Agriculture and Veterinary Medicine, School of Veterinary \\ Medicine, Jimma, Ethiopia
}

\begin{abstract}
Antimicrobial resistance (AMR) is resistance of a microorganism to an antimicrobial that was originally effective for treatment of infections caused by it. Increasing clinical incidence of antimicrobial resistance is a major global health care issue and the situation is perhaps aggravated in developing countries. Although, AMR is a major health care issue, there is a shortage of documented information on it. Therefore, the aim of this paper is to review the causes or risk factors, problems, mechanisms and control of antimicrobial resistance. The resistance problem can be seen simplistically as an equation with two main components: the antibiotic or antimicrobial drug, which inhibits susceptible organisms and selects the resistant ones; and the genetic resistance determinant in microorganisms selected by the antimicrobial drug. Antimicrobial resistance is associated with high mortality rates and high medical costs and has a significant impact on the effectiveness of antimicrobial agents. To appreciate the mechanisms of antimicrobial resistance, it is important to understand how antimicrobial agents act. The resistance mechanisms therefore depend on which specific pathways are inhibited by the drugs and the alternative ways available for those pathways that the organisms can modify to get a way around in order to survive. A comprehensive strategy is necessary to address the challenges that accompany the rising threat of antimicrobial resistance. Special vigilance must now be paid to appropriate selection and timing of antimicrobial agents as a major force in reducing the development of antimicrobial resistance. Prevention and control of these infections will require new antimicrobial agents, prudent use of existing agents, new vaccines, and enhanced public health efforts to reduce transmission.
\end{abstract}

Keywords: Antimicrobial; Health; Mechanism; Microorganism; Resistance; Threat.

Cite This Article: Daba Gudata, and Feyissa Begna. (2018). “ANTIMICROBIAL RESISTANCE: REVIEW." International Journal of Research - Granthaalayah, 6(11), 77-93. https://doi.org/10.29121/granthaalayah.v6.i11.2018.1091.

\section{Introduction}

Microorganisms have existed on the earth for more than 3.8 billion years and exhibit the greatest genetic and metabolic diversity. They are an essential component of the biosphere and serve an important role in the maintenance and sustainability of ecosystems. It is believed that they compose about $50 \%$ of the living biomass. In order to survive, they have evolved mechanisms that enable 
them to respond to selective pressure exerted by various environments and competitive challenges. The disease-causing microorganisms have particularly been vulnerable to man's selfishness for survival who has sought to deprive them of their habitat using antimicrobial agents. These microorganisms have responded by developing antimicrobial drug resistance to fight off this offensive (IDSA, 2011).

The discovery of antimicrobial agents resulted to successful treatment and elimination of infections. Previously fatal diseases were treatable in a significant number of patients (Kharb et al., 2011). Development of antimicrobial agents for clinical use has been most successful in targeting essential components of 5 general areas of microbial metabolism: cell wall synthesis, protein synthesis, RNA synthesis, DNA synthesis and intermediary metabolism (Hooper, 2001). However, discovery of antimicrobial agents have been tempered by the emergence of microbial pathogens that are resistant to antimicrobial agents where by microbial pathogens have developed numerous resistance mechanisms that enable them to evade the effect of antimicrobial agents. As a result, many have become resistant to almost every available antimicrobial agent (Langton et al., 2005).

Currently antimicrobial resistance among bacteria, viruses, parasites, and other disease-causing organisms is a serious threat to infectious disease management globally. The increasing clinical incidence of antimicrobial resistance is a major global health care issue (Ferri et al., 2017) and the situation is perhaps aggravated in developing countries like Ethiopia due to the misuse of antimicrobial, unskilled practitioners, and drug consumers, coupled with rapid spread of resistant microorganisms and inadequate surveillance contributed to antimicrobial resistance (Byarugaba, 2004; Getahun et al., 2013). It is well known that any use of antimicrobials however appropriate and justified, contributes to the development of resistance, but widespread unnecessary and excessive use makes the situation worse (Laxminarayan et al., 2013). Misuse of antimicrobial is facilitated in developing countries by their availability over the counter, without prescription and through unregulated supply chains (Okeke et al., 2005). Noncompliance in the use of antimicrobial has many repercussions upon resistance and poverty is a major root factor of antimicrobial misuse in developing countries (Okeke et al., 2005). On the other hand, even among the rich, some patients miss doses either by mistake or deliberate, especially in cases where signs and symptoms begin to subside after an initial favorable therapeutic response (Hart and Kariuki, 1998).

AMR can lead to increased mortality, morbidity, length of hospital stays, costs of treatment and loss of production in animals. Emerging AMR threatens to undermine the management of microbial infections. Consequences of AMR may be felt harder in resource poor settings as they may be unavailable or unaffordable (Spicknall et al., 2013). Even though antimicrobial resistance is a major global health care issue and the situation is perhaps aggravated in developing countries due to misuse of antimicrobial agent, there is a shortage of documented information on antimicrobial resistance. Therefore, the objective of this paper is to review causes or risk factors, problems, mechanisms and control of antimicrobial resistance. 


\section{Literature Review}

\subsection{Mechanism of Action of Antimicrobial Agents}

In order to appreciate the mechanisms of antimicrobial drug resistance, it is important to understand how antimicrobial agents act. Antimicrobial agents act selectively on vital microbial functions with minimal effects or without affecting host functions. Different antimicrobial agents act in different ways. Better understanding of how antimicrobial agents induce microbial cell death is centered on the essential microbial cell function that is inhibited by the primary drug-target interaction. Antimicrobial agents can be classified based on the cellular component or system they affect, in addition to whether they induce cell death (bactericidal agents) or merely inhibit cell growth (bacteriostatic agents) (Kohanski et al., 2010). Antimicrobial agents act by targeting specific sites of microbes; therefore antimicrobial agents are often categorized according to their principal mechanism of action. Well known mechanisms of action of antimicrobial agents include; interference with cell wall synthesis, inhibition of protein synthesis, interference with nucleic acid synthesis, inhibition of intermediary metabolic pathways and disruption of the cytoplasmic membrane (Tenover, 2006).

\subsubsection{Interference with Microbial Cell Wall Synthesis}

On the basis of the number of antimicrobial drugs in clinical use, microbial cell wall synthesis has been perhaps the target area most extensively exploited for antimicrobial development. The components of the cell wall synthesis machinery are appealing antimicrobial targets because of the absence of counterparts in animal and human biology, thereby providing intrinsic target selectivity (Hooper, 2001).

The most commonly used antimicrobial agents that inhibit cell wall biosynthesis include $\beta$-lactam antibiotics such as penicillin, cephalosporin, carbapenems, oxapenams, and cephamycins (Kotra and Mobashery, 1998). These $\beta$-lactam antibiotics interact and efficiently inhibit the bacterial transpeptidases that catalyze the final cross linking reactions of peptidoglycan synthesis in bacteria directly. The $\beta$-lactams as transpeptidase inhibitors thus block the conversion of immature to mature peptidoglycan therefore these enzymes are often termed penicillin-binding proteins (PBPs). In the presence of the drug, the transpeptidases form a lethal covalent penicilloyl enzyme complex that serves to block the normal transpeptidation reaction. This results in weakly crosslinked peptidoglycan, which makes the growing bacteria highly susceptible to cell lysis and death (Wilke et al., 2005).

The other class of antimicrobial agents that inhibit cell wall synthesis is glycopeptides. The mechanism of action common to all members of the glycopeptides class, including vancomycin, is the inhibition of peptidoglycan synthesis. Vancomycin and other glycopeptides bind to the carboxyl terminal acyl-D-alanyl-D-alanine (acyl-D-Ala-D-Ala) residues of the pentapeptide moiety of lipid II. This specific binding, which is referred to as the primary binding site, sterically hinders the transglycosylase enzyme from incorporating the disaccharide-pentapeptide monomer into nascent peptidoglycan. This inhibition of peptidoglycan synthesis by vancomycin results in a relatively slow inhibition compared with $\beta$-lactams, and its bactericidal activity does not depend on concentration. The transglycosylase enzyme that transfers the disaccharide of the peptidoglycan 
precursor to the growing glycan polymer of the cell wall peptidoglycan is inhibited, presumably due to the steric bulkiness of the glycopeptides peptidoglycan precursor. Both the transglycosylase and transpeptidase enzyme reactions that complete the synthesis of the rigid cell wall peptidoglycan are inhibited by the glycopeptides (Nagarajan, 1991).

\subsubsection{Inhibition of Protein Synthesis}

Macrolides bind to the 50S ribosomal subunit and interfere with the elongation of nascent polypeptide chains. Aminoglycosides inhibit initiation of protein synthesis and bind to the 30S ribosomal subunit (Vannuffel and Cocito, 1996). Chloramphenicol binds to the 50S ribosomal subunit blocking peptidyltransferase reaction. Tetracycline inhibits protein synthesis by binding to 30S subunit of ribosome, thereby weakening the ribosome-tRNA interaction (Reinert et al., 2003). The semi synthetic tetracycline derivatives, colloquially termed the glycylglycines, act at the bacterial ribosome to arrest translation. The glycylglycines bind the ribosome more tightly than previous tetracycline, so that the TetM resistance factor is unable to displace them from this site, hence TetM is unable to protect the ribosome from the action of these new drugs. The TetAmediated efflux system is ineffective against the glycylglycines, as they are not substrates for the transporter. The oxazolidinones, one of the newest classes of antibiotics, interact with the A site of the bacterial ribosome where they should interfere with the placement of the aminoacyl-tRNA (Strohl, 2004; Leach et al., 2007).

\subsubsection{Interference with Nucleic Acid Synthesis}

Inhibitors of DNA topoisomerases: DNA synthesis, mRNA transcription and cell division require the modulation of chromosomal super coiling through topoisomerase-catalyzed strand breakage and rejoining reactions (Kohanski et al., 2010). DNA topoisomerase enzymes are divided into two types, I and II, depending on whether they catalyze reactions involving the transient breakage of one (type I) or both (type II) strands of DNA. Topoisomerases control the topological state of DNA within cells and are critical for the essential processes of protein translation and cell replication (Ehmann and Lahiri, 2014). DNA gyrase, a type II DNA topoisomerase, is the enzyme that negatively super coils DNA in the presence of ATP. In addition, this enzyme plays a role in catenation and decatenation reaction of a double-stranded DNA circles, resolves knots in DNA and also relaxes negatively super coiled DNA in the absence of ATP. As a result, the enzyme is vital for almost all cellular processes that involve duplex DNA, namely replication, recombination and transcription. It is exclusive to the prokaryotic kingdom and is essentially crucial for the survival of the organism. Thus, DNA gyrase remain an ideal and attractive target for antibacterial drugs (Chatterji et al., 2001).

Quinolones are the most successful antimicrobial agents targeting to DNA gyrase. The compounds originated from nalidixic acid, a naphthyridone discovered by accident as a by-product during the synthesis of chloroquine (Collin et al., 2011). Quinolones are specific inhibitors of DNA gyrase. Quinolones inhibit reactions of DNA gyrase such as super coiling and relaxation that require DNA breakage and re-union; specifically they interfere with the breakage-re-union reaction of DNA gyrase by interacting with subunit A (GyrA) (Chen and Liu, 1994). The first-generation quinolones, nalidixic acid and oxolinic acid have relatively weak antimicrobial activity. However, the synthesis of fluoroquinolones and their improvement over several generations, e.g., norfloxacin 
and ciprofloxacin (second gen.), levofloxacin (third gen.) and moxifloxacin and gemifloxacin (fourth gen.), has led to a range of potent antimicrobial agents (Collin et al., 2011). Besides the type II topoisomerases, most bacterial pathogens possess an additional essential topoisomerase, topoisomerase I (Topo I). Topo I is architecturally and mechanistically distinct from gyrase and topoisomerase IV and as such represents an attractive target for the discovery of novel antibacterial chemotypes (Ehmann and Lahiri, 2014).

Inhibitors of microbial RNA synthesis: Rifamycins inhibit DNA-dependent transcription by binding with high affinity to the $\beta$-subunit (encoded by rpoB) of a DNA-bound and actively transcribing RNA polymerase. The $\beta$-subunit is located in the channel that is formed by the RNA polymerase-DNA complex, from which the newly synthesized RNA strand emerges. Rifamycins uniquely require RNA synthesis to not have progressed beyond the addition of two ribonucleotides; this is attributed to the ability of the drug molecule to sterically inhibit nascent RNA strand initialization. It is worth noting that rifamycins are not thought to act by blocking the elongation step of RNA synthesis, although a recently discovered class of RNA polymerase inhibitors could inhibits elongation by allosterically modifying the enzyme (Kohanski et al., 2010).

\subsubsection{Inhibition of Microbial Metabolic Pathways}

Trimethoprim and sulfonamides interfere with folic acid metabolism in the microbial cell by competitively blocking the biosynthesis of tetrahydrofolate, which acts as a carrier of one-carbon fragments and is necessary for the ultimate synthesis of DNA, RNA and bacterial cell wall proteins. Unlike mammals, bacteria and protozoan parasites usually lack a transport system to take up preformed folic acid from their environment. Most of these organisms must synthesize folic acid, although some are capable of using exogenous thymidine, circumventing the need for folic acid metabolism (Džidic et al., 2008).

Sulfonamides competitively inhibit the conversion of pteridine and para-aminobenzoic acid (PABA) to dihydrofolic acid by the enzyme pteridine synthetase. Sulfonamides have a greater affinity than PABA for pteridine synthetase. Trimethoprim has a tremendous affinity for bacterial dihydrofolate reductase (10,000 to 100,000 times higher than for the mammalian enzyme); when bound to this enzyme, it inhibits the synthesis of tetrahydrofolate (Baron, 1996).

\subsubsection{Disruption and Increased Permeability of Cytoplasmic Membrane}

Biologic membranes are composed basically of lipids, proteins and lipoproteins. The cytoplasmic membrane acts as a diffusion barrier for water, ions, nutrients and transport systems. Most health workers now believe that membranes are a lipid matrix with globular proteins randomly distributed to penetrate through the lipid bilayer. A number of antimicrobial agents can cause disorganization of the membrane. These agents can be divided into cationic, anionic, and neutral agents. The bestknown compounds are polymyxin B and colistemethate (polymyxin E) (Džidic et al., 2008).

Cytoplasmic membrane forms an effective barrier to many antimicrobial agents. The mode of action of some antimicrobial agents may lie in the ability of certain drugs to increase the permeability of the membrane, facilitating the entry of themselves and other compounds. Cationic antibacterial agents, such as polymyxin B have been reported to increase the permeability of the 
outer membrane to lysozyme and hydrophobic compounds. The initial action of these antimicrobial agents is to disrupt outer membrane structure, allowing themselves and other compounds to enter the cell and inhibit specific metabolic processes (Baron, 1996). Polymixin B has several cell-damaging properties: (i) it disturbs the surface charge, lipid composition and structure of the membranes; (ii) it dissipates the $\mathrm{K}+$ gradient on the cytoplasmic membrane; and (iii) it depolarizes the cytoplasmic membrane. The permeability of the outer membrane to lipophilic compounds is one of the main factors controlling bacterial sensitivity to polymixin B. Since polymixin B is bulkier than the inorganic divalent cations it displaces, the packing order of lipopolysaccharide (LPS) is altered in the presence of polymixin B. This results in increased permeability of the outer membrane to a variety of molecules and also facilitates the uptake of polymixin B ("self-promoted" uptake) (Krupovivc et al., 2007).

\subsection{Causes or Risk factors of Antimicrobial Resistance}

The resistance problem can be seen simplistically as an equation with two main components: the antibiotic or antimicrobial drug, which inhibits susceptible organisms and selects the resistant ones; and the genetic resistance determinant in microorganisms selected by the antimicrobial drug (Levy, 2002). Drug resistance emerges only when the two components come together in an environment or host, which can lead to a clinical problem. Selected resistance genes and their hosts spread and propagate under continued antimicrobial selection to amplify and extend the problem to other hosts and other geographic locations. Millions of kilograms of antimicrobials are used each year in the prophylaxis and treatment of people, animals and agriculture globally (Levy and Marshall, 2004), driving the resistance problem by killing susceptible strains and selecting those that are resistant.

The situation is perhaps aggravated in developing countries like Ethiopia due to the misuse of antimicrobials, unskilled practitioners, and drug consumers, coupled with rapid spread of resistant microorganisms and inadequate surveillance contributed to antimicrobial drug resistance (Byarugaba, 2004; Getahun et al., 2013). It is well known that any use of antimicrobials however appropriate and justified, contributes to the development of resistance, but widespread unnecessary and excessive use makes the situation worse (Laxminarayan et al., 2013).

A number of important factors that include microbial characteristics, environmental or human reservoirs in which resistance genes or resistant organisms can persist, patterns of antimicrobial use, and societal and technologic changes that affect the transmission of organisms. Various combinations of these factors determine the frequency of occurrence of drug-resistant organisms. Certain microbial characteristics can apparently affect the frequency of antimicrobial resistance by enhancing the organism's ability to emerge, persist, or be transmitted. Such characteristics include the propensity to easily exchange genetic material, possess intrinsic resistance, survive varying environmental conditions, occupy certain ecologic niches, easily colonize, and infect (Baharoglu et al., 2013).

\subsection{Problems Associated with Antimicrobial Resistance}

Antimicrobial resistance is associated with high mortality rates and high medical costs and has a significant impact on the effectiveness of antimicrobial agents. Antimicrobial resistance provokes 
obstruction in disease control by intensifying the possibility of spreading of resistant pathogens, thus, declining efficacy of treatment and, hence, resulting in prolonged time of infection in patient. The cost of treatment is also increased due to antimicrobial drug resistance as the pathogens have become resistant to commercially available drugs, which has triggered the use of more expensive therapies (Tanwar et al., 2014). The rate of success of present day medical applications like organ transplantation and cancer chemotherapy has contributed immensely towards development of drug resistance. Differences in the resistance profiles of bacterial and fungal pathogens as well as the quality of public hygiene also have a considerable impact on the effectiveness of antimicrobial agents. Expansion of global trade and tourism lead to increased potential of antimicrobial resistance to spread all over the world and decrease in export and import of various products affecting the economy of developing countries (Tanwar et al., 2014).

\subsection{Classification of Antimicrobial Resistance}

Despite of administration of appropriate doses of drugs for a specific duration of time, survival of various microbial strains recommends the high levels of resistance developed in them. This clinical failure is due to not only the antimicrobial resistance but also the suppressed immune function, poor/deprived drug bioavailability, or increased rate of drug metabolism. Persistence of microbes after conventional/standard treatments points out different types of antimicrobial resistance which is an expanding problem in medical world. Antimicrobial resistance can be classified as intrinsic, circumstantial and acquired resistance (Adekunle, 2012).

\subsubsection{Intrinsic resistance}

Intrinsic resistance to an antimicrobial agents characterizes resistance that is an inherent attribute of a particular species; these organisms may lack the appropriate drug- susceptibility targets or posses natural barriers that prevent the agents from reaching the target; examples are the natural resistance of gram negative bacteria to vancomycin because of the drug's inability to penetrate the gram-negative outer membrane, or the intrinsic resistance of the penicillin (Bozdogan et al., 2003; Loeffler and Stevens, 2003).

\subsubsection{Circumstantial Resistance}

Circumstantial resistance is the difference between the in vitro and in vivo effects of an antimicrobial agent. Agents that appear to be active in the laboratory may be ineffective in vivo because of failure to reach the site of infection, such as the inability of first-generation cephalosporin to cross the blood-brain barrier. Drugs such as aminoglycosides may be inactivated; in vivo antagonist of trimethoprim sulfamethoxazole can be overcome by enterococci via their inability to take up and internalize corporate environmental folate (Hidron et al., 2008).

\subsubsection{Acquired or Active Resistance}

Acquired resistance is the major mechanism of antimicrobial resistance, is the result of a specific evolutionary pressure to develop a counterattack mechanism against an antimicrobial or class of antimicrobials so that bacterial populations previously sensitive to antimicrobials become resistant. This type of resistance results from changes in the bacterial genome. Resistance in 
bacteria may be acquired by a mutation and passed vertically by selection to daughter cells. More commonly, resistance is acquired by horizontal transfer of resistance genes between strains and species. Exchange of genes is possible by transformation, transduction or conjugation (Langton et al., 2005; Yoneyama and Katsumata, 2006).

\subsection{Genetics of Antimicrobial Resistance}

Antimicrobial resistance can be acquired from (i) mutation of cellular genes (ii) horizontal gene transfer through conjugation, transformation and transduction, and (iii) a combination of these mechanisms (Raghunath, 2008; Adhikari, 2010).

\subsubsection{Mutations}

Spontaneous mutations: Chromosomal mutations are quite rare (one in a population of $10^{6}-10^{8}$ microorganisms) and commonly determine resistance to structurally related compounds (Adhikari, 2010). These mutations occur as errors of replication or an incorrect repair of damaged DNA. They are called spontaneous mutations or growth-dependent mutations. Resistance to quinolones in $E$. coli is caused by changes in at least seven amino acids in the gyrA gene or three amino acids in the parC gene (Džidic et al., 2008), whereas only a single point mutation in the rpoB gene is associated with a complete resistance to rifampin (Adhikari, 2010). A chromosomal mutation in dihydropteroate synthetase results in a reduced affinity for sulfonamides. Some biochemical resistance mechanisms are the result of mutations. Antibiotic uptake or efflux system can be modified by mutation (Hooper, 2001).

Hypermutators: According to the "hypermutable state" model, a small bacterial population during a prolonged nonlethal selection of microorganisms may achieve a short-term state when the population mutates at a very high rate (hyper mutable strains or mutators) (Džidic et al., 2008). These cells can increase the rate of mutations from 10 to 50 up to 10000 times (Martinez and Baquero, 2000). Most hypermutators are found in populations of E. coli, Salmonella enterica, Neisseria meningitides (N. meningitides), Haemophilus influenzae, Staphilococcus aureus, Helicobacter pylori (H. pylori), Streptococcus pneumoniae (S. pneumoniae), and P. aeruginosa (Džidic et al., 2008).

Adaptive mutagenesis: Most mutations occur in dividing cells (spontaneous mutation). However, they can also arise in non dividing or slowly dividing cells (adaptive mutation). Mutations occur only during nonlethal selection of microorganisms and are called "adaptive mutations." This adaptive process is the only and main source of the antibiotic-resistant mutants to originate under normal conditions. Streptomycin causes a hypermutable phenotype in E. coli, and some antibiotics (quinolones) can induce the mutagenic response and increase the rate of emergence of resistance to antibiotics (Erill et al., 2006).

\subsubsection{Horizontal Gene Transfer}

A transfer of resistance genes from one bacterium to another is called a horizontal gene transfer (Bennett, 2008). Genes can be transferred by three main ways: transduction (via bacteriophages and integrons), conjugation (via plasmids and conjugative transposons), and transformation (via incorporation of chromosomal DNA, plasmids into a chromosome). Then genes are incorporated 
into the recipient chromosome by recombination or transposition and may have one or several changes in gene sequence (Alekshun and Levy, 2007; Hawkey, 2008).

Most plasmids are double-stranded circular DNA whose size may vary from 2-3 kb to plasmids, which encode up to $10 \%$ of the host cell chromosome. The transfer of resistance genes is more effective than chromosomal mutation (Alekshun and Levy, 2007). Plasmids encode genes that confer resistance to main classes of antimicrobial agents (cephalosporin, fluoroquinolones, and aminoglycosides) (Bennett, 2008), toxic heavy metals (mercury, cadmium, silver), and virulence determinants that help a cell to survive in the environment of lethal antibiotic doses (Hawkey, 2008). Resistance determinants gene can be transferred in one of the following three ways (Langton et al., 2005; Adekunle, 2012).

Transformation: is the simplest type of gene transfer. A recipient cell acquires genes from 'free floating' DNA molecules in the surrounding medium. In nature, the DNA may come from dead cells that lyses and release their DNA. In the laboratory, however, the DNA is extracted by chemical methods from a suspension of donor bacteria and then added to a culture of recipient bacteria. In nature or in the laboratory, a recipient bacterium can acquire one or more inheritable characteristics from a donor bacterium and become what is called transformed. Only certain species of bacteria are known to undergo transformation, and even these must be in a state of growth receptive to the incorporation of donor DNA; that is they must be competent. This condition usually occurs when the recipient bacteria are in the late logarithm phase of their growth. Competent bacteria cells produce a special protein that binds donor DNA fragments at specific sites on the cell surface. Although chromosomal DNA can be readily transferred to competent recipient bacteria, plasmid DNA is not easily transferred by ordinary transformation procedure that simply add DNA to recipient cells. However, special procedures widely used in genetic engineering can be used to accomplish transformation with plasmid DNA (Adekunle, 2012).

Transduction: is gene transfer in which a virus serves as the vehicle for carrying DNA from a donor bacterium to a recipient bacterium. A phage consists of a nucleic acid, usually DNA surrounded by a protein coat to form a head. A tail like appendage serves to attach the phage to the surface of a susceptible host bacterium. After the phage injects its DNA into the host cell, the phage DNA is replicated rapidly while the bacterial DNA is degraded. The phage DNA then directs the synthesis of new phage proteins by the host cell. Within a short time the new phage, DNA molecules combine the new phage proteins to form numerous whole phages, which are released as the host cell disintegrates. During assembly of the phage progeny within the infected host cell, any fragment of the host bacterium's DNA that is approximately the same size as the phage DNA may be accidentally incorporated into a new phage head instead of the phage DNA. A phage carrying such a fragment is called a transducting phage because if it affects another bacterium, it injects the bacterial DNA fragments into the new host. Because the transducting phages do not contain the entire viral DNA, they do not kill the new host. The fragment can then undergo recombination with the corresponding part of the new host's chromosome and become a permanent part of that chromosome. Thus, the second bacterial host acquires one or more genes (Yoneyama and Katsumata, 2006; Adekunle, 2012).

Conjugation: is a process of gene transfer that requires cell to cell contact. Plasmids also are capable of horizontal transfer via conjugation, although the efficiency of plasmid transfer both within and between species can vary tremendously. DNA may be transferred directly from one bacterium to another. Bacterial conjugation differs from sexual mating in eukaryotes in that it does 
not involve the fusion of two gametes to form a single cell. In some types of conjugation, only a plasmid may be transferred from the donor bacterium to the recipient bacterium. In other types, large segments of the donor cell's chromosome or even the entire chromosome may be transferred to a recipient's cell. This differs from transformation and transduction, in which only relatively small chromosomal fragments may be, transferred (Langton et al., 2005; Adekunle, 2012).

\subsection{Mechanisms of Antimicrobial Resistance}

Prior to the 1990s, the problem of antimicrobial resistance was never taken to be such a threat to the management of infectious diseases. But gradually treatment failures were increasingly being seen in health care settings against first-line drugs and second-line drugs or more. Microorganisms were increasingly becoming resistant to ensure their survival against the arsenal of antimicrobial agents to which they were being bombarded. They achieved this through different means but primarily based on the chemical structure of the antimicrobial agent and the mechanisms through which the agents acted. The resistance mechanisms therefore depend on which specific pathways are inhibited by the drugs and the alternative ways available for those pathways that the organisms can modify to get a way around in order to survive (Cohen, 2000).

Mechanisms for acquired resistance (Langton et al., 2005; Donkor and Badoe, 2014).

- The presence of an enzyme that inactivates the antimicrobial agent

- The presence of an alternative enzyme for the enzyme that is inhibited by the antimicrobial agent

- A mutation in the antimicrobial agent's target, which reduces the binding of the antimicrobial agent

- Post-transcriptional or post-translational modification of the antimicrobial agent's target, which reduces binding of the antimicrobial agent

- Reduced uptake of the antimicrobial agent

- Active efflux of the antimicrobial agent

- Overproduction of the target of the antimicrobial agent

- Expression or suppression of a gene in vivo in contrast to the situation invitro

- Previously unrecognized mechanisms.

Although the manner of acquisition of resistance may vary among microbes, resistance is created by only a few mechanisms (Džidić et al., 2008):

- Antibiotic inactivation: - direct inactivation of the active antibiotic molecule (Wright, 2005);

- Target modification: - alteration of the sensitivity to the antibiotic by modification of the target (Lambert, 2005);

- Efflux pumps and outer membrane (OM) permeability changes: - reduction of the concentration of drug without modification of the compound itself (Kumar and Schweizer, 2005); or

- Target bypass: - some bacteria become refractory to specific antibiotics by bypassing the inactivation of a given enzyme (Happi et al., 2005; Džidić et al., 2008). 


\subsubsection{Drug Inactivation}

The defense mechanisms within the category of antibiotic inactivation include the production of enzymes that degrade or modify the drug itself. The major enzymes that inactivate antimicrobial agents include; $\beta$-lactamases, aminoglycoside-modifying enzymes and chloramphenicol acetyltransferases (Giedraitiene et al., 2011). Unlike other mechanisms, inactivation of antimicrobial agents confers resistance to structurally related drugs. Biochemical strategies are hydrolysis, group transfer, and redox mechanisms (Džidić et al., 2008).

Antimicrobial inactivation by hydrolysis: Many antibiotics have hydrolytically susceptible chemical bonds (e.g. esters and amides). Several enzymes are known to destroy antibiotic activity by targeting and cleaving these bonds (Hunter et al., 2008). Genes that encode $\beta$-lactamases are transferred by transposons but also they may be found in the composition of integrons (Jacoby and Munoz-Price, 2005). The classical hydrolytic amidases are the $\beta$-lactamases that cleave the $\beta$ lactam ring of the penicillin and cephalosporin antibiotics.

Drug inactivation by group transfer: The group of enzymes inactivating aminoglycosides, chloramphenicol, streptogramin, macrolides or rifampicin is called transferases. Inactivation is made by binding adenyl, phosphoryl, or acetyl groups to the periphery of the drug molecule. These modifications are achieved in the process of transport across the cytoplasmic membrane (Džidic et al., 2008).

Drug inactivation by redox process: Oxidation and reduction reactions are used by pathogenic organisms as a resistance mechanism against antimicrobial. Streptomyces virginiae produces type A antibiotic virginiamycin and protects itself from its own antimicrobial agents by substituting a ketone group to an alcohol residue (Džidic et al., 2008).

\subsubsection{Drug Resistance by Target Site Modification}

The second major resistance mechanism is the modification of the antibiotic target site so that the antibiotic is unable to bind properly (Džidic et al., 2008). An interaction between an antimicrobial agent and a target molecule is very specific so even small changes in a target molecule can influence antibiotic binding to a target (Giedraitiene et al., 2011). Antimicrobial agents act at targets that are present in microbial cells but differ sufficiently to mammalian cells to allow for selective inhibition of the bacterial counterparts. Because of the vital cellular functions of the target sites, organisms cannot evade antimicrobial action by dispensing with them entirely. However, microbes have been acquiring some mutational changes in the target that reduce susceptibility to inhibition whilst retaining their cellular function (Lambert, 2002). Examples of resistance due to target alterations are discussed below.

Alterations of Penicillin Binding Proteins: Mosaic PBPs in Streptococcus pneumonia; Resistance to $\beta$-lactam antimicrobial agents in Streptococcus pneumoniae is due to the development of penicillin binding proteins (PBPs) with decreased affinity for the drugs. Resistance to third-generation cephalosporins is due to the presence of altered forms of PBP1a and 2x, whereas penicillin resistance also involves alterations in PBP2b (Nagai et al., 2002; Kosowska et al., 2004). The altered PBPs are generated by recombinational events between the PBP genes of $S$. pneumoniae and related PBP genes from closely related streptococcal species acquired by transformation of this naturally competent organism (Coffey et al., 1995). 
Modification of peptidoglycan precursors: The cause of resistance to the glycopeptides antibacterial agents in Enterococcus faecium and Enterococcus faecalis is the acquisition of one of two related gene clusters, known as VanA and VanB. These gene clusters encode enzymes that produce a modified peptidoglycan precursor terminating in D-Alanyl-D-Lactate (D-Ala-D-Lac) instead of D-Ala-D-Ala (Bugg et al., 1991). The glycopeptides bind with much lower affinity to D-Ala-D-Lac than to D-Ala-D-Ala (Cooper et al., 2000). The low affinity binding of glycopeptides to D-Ala-D-Lac results from an altered pattern of intermolecular hydrogen bonding responsible for the normally high affinity binding between drug and its substrate (Williams et al., 1998).

Modification by ribosomal methylation: So far, ribosomal methylation remains the most widespread mode of resistance to macrolides and lincosamides (Weisblum, 1995). As a consequence of methylation, binding of erythromycin to its target is impaired. The overlapping binding sites of macrolides, lincosamides and streptogramins B in $23 \mathrm{~S}$ rRNA, account for crossresistance to the 3 classes of drugs (Vannuffel et al., 1992; Wang and Taylor, 1998). A wide range of microbial pathogens that are targets for macrolides and lincosamides, including gram-positive species, spirochetes and anaerobes, express Erm methylases. Nearly 40 erm genes have been reported so far (Roberts et al., 1999).

Interference with DNA synthesis: The mechanism of resistance is a modification of two enzymes: DNA gyrase (also known as topoisomerase II) (genes gyrA and gyrB) (Kim et al., 2002) and topoisomerase IV (parC and parE). Mutations in genes gyrA and parC are followed by replication failure, and then quinolones/fluoroquinolones cannot bind. The most common mutation in E. coli gyrA causes a reduced drug affinity for modified DNA complex, and MIC is higher. Quinolones (ciprofloxacin) bind to DNA gyrase A subunit. Usually resistance to quinolones is associated with mutations in chromosomes, but plasmid mediated and point mutation-related (in genes gyrA and parC) resistance to quinolones was reported as well (Giedraitiene et al., 2011).

\subsubsection{Active Drug Efflux}

Efflux pumps are energy-dependent transporters that extrude toxic compounds, including antimicrobials, being one of the major mechanisms by which microbial pathogens resist to different classes of antimicrobial agents (Guilhelmelli et al., 2013). The efflux systems are composed of three protein components, an energy-dependent pump located in the cytoplasmic membrane, an outer membrane porin and a linker protein which couples the two membrane components together (Nikaido, 1998). Efflux pumps can be specific to antimicrobials or may be capable to pump a wide range of unrelated agents e.g. macrolides, tetracycline and fluoroquinolones and thus significantly contribute to multidrug resistance (MDR) (McManus, 1997). Active efflux of drugs from the cell is one of the common mechanisms of antimicrobials resistance in bacteria, with resistance developing when the rate of drug efflux across the membrane exceeds that of drug influx, bacterial genomes encode several membrane-bound multidrug efflux systems. These systems are usually under the control of an intricate regulatory network, which, in response to the presence of drug and other stress molecules, increases the overall efflux activity and decreases influx capacity (Misra et al., 2015). Microbial pathogens resistant to tetracycline often produce increased amounts of membrane proteins that are used as export or efflux pumps of antimicrobial agents. To eliminate toxic compounds from the cytoplasm and periplasm, $P$. aeruginosa uses more than four powerful multidrug resistant efflux pumps. Multidrug resistance efflux pumps are responsible for resistance to fluoroquinolones, tetracycline, chloramphenicol and erythromycin (Giedraitiene et al., 2011). 


\subsubsection{Reduced Membrane Permeability}

The outer membrane in gram-negative organisms contains an inner layer that has phospholipids and an outer layer that has the lipid A. Such composition reduces drug uptake to a cell and transfer through the outer membrane (through porin proteins, e.g., OmpF in E. coli and OprD in $P$. aeruginosa). Drug molecules to a cell can be transferred by the following mechanisms: (i) diffusion through porins, (ii) diffusion through the bilayer, and (iii) by self-promoted uptake. Acquired resistance to all antimicrobial classes in P. aeruginosa is due to low outer membrane permeability. Small hydrophilic molecules ( $\beta$-lactams and quinolones) can cross the outer membrane only through porins. Amino glycosides and colistin cannot be transferred to the cell through porins; therefore, self-promoted uptake to the cell is initiated by binding to lipopolysaccharides of the outer side of the outer membrane (Labert, 2002). Acquired resistance is characteristic of high resistance to almost all aminoglycosides (especially to tobramycin, netilmicin, and gentamicin) (Ferguson et al., 2007).

\subsubsection{Target Bypass}

Some bacteria become refractory to specific antibiotics by bypassing the inactivation of a given enzyme. This mode of resistance is observed in many trimethoprim and sulfonamide-resistant bacteria. The example is in bypassing inhibition of dihydrofolate reductase (DHFR) and dihydropteroate synthase (DHPS) enzymes (involved in tetrahydrofolate biosynthesis). They are inhibited by trimethoprim and sulfonamides, respectively. In several trimethoprim and sulfonamide-resistant strains, a second enzyme that has low affinity for the inhibitors is produced (Džidić et al., 2008; Happi et al., 2005).

\subsection{Control and Prevention of Antimicrobial Resistance}

A comprehensive strategy is necessary to address the challenges that accompany the rising threat of AMR. The Transatlantic Task Force on antimicrobial resistance (TATFAR) outlined three critical tasks that must be undertaken to effectively fight. First, therapeutic use of antimicrobials needs to be conducted appropriately in medical and veterinary contexts. Second, drug-resistant infections need to be controlled and prevented. Third, strategies are necessary to preserve existing antimicrobials and improve the development pipeline for new antimicrobial agents, alternative therapies and diagnostic devices (Paphitou, 2013).

Controlling microbial resistance to antimicrobial agents requires a multifaceted approach. Essential components of the approach must include reducing inappropriate prescribing not only for humans but also for animals, reducing transmission of resistant organisms through enhanced infection control and environmental hygiene, and identifying trends in resistance through surveillance. This approach fits neatly within the classic bug-drug-host paradigm. The overuse of antibiotics is considered the main factor in the emergence and dissemination of antimicrobial resistance (Conly, 2002). Special vigilance must now be paid to appropriate selection and timing of antimicrobial agents as a major force in reducing the development of antimicrobial resistance. Proper hygiene practices will help to reduce plasmid transfer and the establishment of multiple drug-resistant microbial pathogens in the hospital and will delay the appearance of such species in 
the community. The health care provider must be continually alert to the appearance of drug resistance within the hospital and community (Baron, 1996).

\section{Conclusion and Recommendations}

It is clear that antimicrobial drug resistance is becoming a lethal problem and posing a great threat to our health care system. Antimicrobial Resistance can lead to increased mortality, morbidity, length of hospital stays, costs of treatment and loss of production in animals. Prevention and control of these infections will require new antimicrobial agents, prudent use of existing agents, new vaccines, and enhanced public health efforts to reduce transmission. It is well known that any use of antimicrobials however appropriate and justified, contributes to the development of resistance, but widespread unnecessary and excessive use makes the situation worse. As a rule, rational use of antimicrobial at every level is the key to limit the spread of the resistance. Therefore based on the above information, the following recommendations are forwarded:

- Governments should develop and implement medicine policies and strategies that take into consideration the threat of antimicrobial drug resistance so as to limit the evolution and possible spread of resistant microbial.

- Health worker should give attention to antimicrobial resistance while treating infectious disease with antimicrobial agent.

\section{References}

[1] Adekunle, O.O., 2012. Mechanisms of antimicrobial resistance in bacteria, general approach. International Journal of Pharma Medicine and Biological Sciences, 1(2): 166-187.

[2] Adhikari, L., 2010. High-level aminoglycoside resistance and reduced susceptibility to vancomycin in nosocomial enterococci. Journal of global infectious diseases, 2(3): 231.

[3] Alekshun, M.N. and Levy, S.B., 2007. Molecular mechanisms of antibacterial multidrug resistance. Cell, 128(6): 1037-1050.

[4] Baharoglu, Z., Garriss, G., Mazel, D., 2013. Multiple pathways of genome plasticity leading to development of antibiotic resistance. Antibiotics, 2(2): 288-315.

[5] Baron, S., 1996. Protozoa: Structure, Classification, Growth, and Development Medical Microbiology. University of Texas Medical Branch at Galveston.

[6] Bennett, P.M., 2008. Plasmid encoded antibiotic resistance: acquisition and transfer of antibiotic resistance genes in bacteria. British journal of pharmacology, 153(1): 347-357.

[7] Bozdogan, B., Esel, D., Whitener, C., Browne, F.A., Appelbaum, P.C., 2003. Antibacterial susceptibility of a vancomycin-resistant Staphylococcus aureus strain isolated at the Hershey Medical Center. Journal of Antimicrobial Chemotherapy, 52(5): 864-868.

[8] Bugg, T.D., Wright, G.D., Dutka-Malen, S., Arthur, M., Courvalin, P., Walsh, C.T., 1991. Molecular basis for vancomycin resistance in Enterococcus faecium BM4147: biosynthesis of a depsipeptide peptidoglycan precursor by vancomycin resistance proteins VanH and VanA. Biochemistry, 30(43): 10408-15.

[9] Byarugaba, D.K., 2004. Antimicrobial resistance in developing countries and responsible risk factors. International journal of antimicrobial agents, 24(2): 105-110.

[10] Chatterji, M., Unniraman, S., Mahadevan, S., Nagaraja, V., 2001. Effect of different classes of inhibitors on DNA gyrase from Mycobacterium smegmatis. Journal of Antimicrobial Chemotherapy, 48(4): 479-485.

[11] Chen, A.Y. and Liu, L.F., 1994. DNA topoisomerases: essential enzymes and lethal targets. Annual review of pharmacology and toxicology, 34(1): 191-218. 
[12] Coffey, T.J., Dowson, C.G., Daniels M., Spratt, B.G., 1995. Genetics and molecular biology of $\beta$ lactam-resistant pneumococci. Microbial Drug Resistance, 1(1): 29-34.

[13] Cohen, M.L., 2000. Changing patterns of infectious disease. Nature, 406(6797): 762.

[14] Collin, F., Karkare, S., Maxwell, A., 2011. Exploiting bacterial DNA gyrase as a drug target: current state and perspectives. Applied Microbiology and Biotechnology, 92(3): 479-497.

[15] Conly, J., 2002. Antimicrobial resistance in Canada. Canadian Medical Association Journal, 167(8): 885-891.

[16] Cooper, M.A., Fiorini, M.T., Abell, C., Williams, D.H., 2000. Binding of vancomycin group antibiotics to D-alanine and D-lactate presenting self-assembled monolayers. Bioorganic \& medicinal chemistry, 8(11): 2609-2616.

[17] Donkor, E.S. and Badoe, E.V., 2014. Insights into pneumococcal pathogenesis and antibiotic resistance. Advances in Microbiology, 4(10): 627.

[18] Džidić, S., Šušković, J., Kos, B., 2008. Antibiotic resistance mechanisms in bacteria: biochemical and genetic aspects. Food Technology \& Biotechnology, 46(1):11-21.

[19] Ehmann, D.E. and Lahiri, S.D., 2014. Novel compounds targeting bacterial DNA topoisomerase/DNA gyrase. Current opinion in pharmacology, 18: 76-83.

[20] Erill, I., Campoy, S., Mazon, G., Barbe, J., 2006. Dispersal and regulation of an adaptive mutagenesis cassette in the bacteria domain. Nucleic acids research, 34(1): 66-77.

[21] Ferguson, D., Cahill, O.J., Quilty, B., 2007. Phenotypic, molecular and antibiotic resistance profiling of nosocomial Pseudomonas aeruginosa strains isolated from two Irish Hospitals. Journal of Medical and Biological Science, 1(1): 1-15.

[22] Ferri, M., Ranucci, E., Romagnoli, P., Giaccone, V., 2017. Antimicrobial resistance: a global emerging threat to public health systems. Critical reviews in food science and nutrition, 57(13): 2857-2876.

[23] Getahun, B., Ameni, G., Medhin, G., Biadgilign, S., 2013. Treatment outcome of tuberculosis patients under directly observed treatment in Addis Ababa, Ethiopia. The Brazilian Journal of Infectious Diseases, 17(5): 521-528.

[24] Giedraitienè, A., Vitkauskienè, A., Naginienè, R., Pavilonis, A., 2011. Antibiotic resistance mechanisms of clinically important bacteria. Medicina, 47(3): 19.

[25] Guilhelmelli, F., Vilela, N., Albuquerque, P., Derengowski, L., Silva-Pereira, I., Kyaw, C., 2013. Antibiotic development challenges: the various mechanisms of action of antimicrobial peptides and of bacterial resistance. Frontiers in microbiology, 4: 353.

[26] Happi, C.T., Gbotosho, G.O., Folarin, O.A., Akinboye, D.O., Yusuf, B.O., Ebong, O.O., Sowunmi, A., Kyle, D.E., Milhous, W., Wirth, D.F., Oduola, A.M.J., 2005. Polymorphisms in Plasmodium falciparum dhfr and dhps genes and age related in vivo sulfadoxine-pyrimethamine resistance in malaria-infected patients from Nigeria. Acta tropica, 95(3): 183-193.

[27] Hart, C.A. and Kariuki, S., 1998. Antimicrobial resistance in developing countries. BMJ: British Medical Journal, 317(7159): 647.

[28] Hawkey, P.M., 2008. Molecular epidemiology of clinically significant antibiotic resistance genes. British journal of pharmacology, 153(1): 406-413.

[29] Hidron, A.I., Edwards, J.R., Patel, J., Horan, T.C., Sievert, D.M., Pollock, D.A., Fridkin, S.K., 2008. Antimicrobial-resistant pathogens associated with healthcare-associated infections: annual summary of data reported to the National Healthcare Safety Network at the Centers for Disease Control and Prevention, 2006-2007. Infection Control \& Hospital Epidemiology, 29(11): 9961011.

[30] Hooper, D.C., 2001. Mechanisms of action of antimicrobials: focus on fluoroquinolones. Clinical Infectious Diseases, 32(1): 9-15.

[31] Hunter, P.R., Wilkinson, D.C., Catling, L.A., Barker, G.C., 2008. Meta-analysis of experimental data concerning antimicrobial resistance gene transfer rates during conjugation. Applied and environmental microbiology, 74(19): 6085-90. 
[32] Infectious Diseases Society of America (IDSA), 2011. Combating antimicrobial resistance: policy recommendations to save lives. Clinical Infectious Diseases, 52(5): 397-428.

[33] Jacoby, G.A. and Munoz-Price, L.S., 2005. The new $\beta$-lactamases. New England Journal of Medicine, 352(4): 380-391.

[34] Kharb, R., Shahar Yar, M., C Sharma, P., 2011. New insights into chemistry and anti-infective potential of triazole scaffold. Current medicinal chemistry, 18(21): 3265-3297.

[35] Kim, Y.H., Cha, C.J. and Cerniglia, C.E., 2002. Purification and characterization of an erythromycin esterase from an erythromycin-resistant Pseudomonas sp. FEMS microbiology letters, 210(2): 239-244.

[36] Kohanski, M.A., Dwyer, D.J., Collins, J.J., 2010. How antibiotics kill bacteria: from targets to networks. Nature Reviews Microbiology, 8(6): 423.

[37] Kosowska, K., Jacobs, M.R., Bajaksouzian, S., Koeth, L., Appelbaum, P.C., 2004. Alterations of penicillin-binding proteins $1 \mathrm{~A}, 2 \mathrm{X}$, and $2 \mathrm{~B}$ in Streptococcus pneumoniae isolates for which amoxicillin MICs are higher than penicillin MICs. Antimicrobial agents and chemotherapy, 48(10): 4020-4022.

[38] Kotra, L.P. and Mobashery, S., 1998. $\beta$-Lactam antibiotics, $\beta$-lactamases and bacterial resistance. Bulletin de l'Institut Pasteur, 96(3), pp.139-150.

[39] Krupovič, M., Daugelavičius, R., Bamford, D.H., 2007. Polymyxin B induces lysis of marine pseudoalteromonads. Antimicrobial agents and chemotherapy, 51(11): 3908-3914.

[40] Kumar, A. and Schweizer, H.P., 2005. Bacterial resistance to antibiotics: active efflux and reduced uptake. Advanced drug delivery reviews, 57(10): 1486-1513.

[41] Lambert, P.A., 2002. Mechanisms of antibiotic resistance in Pseudomonas aeruginosa. Journal of the royal society of medicine, 95(41):22.

[42] Lambert, P.A., 2005. Bacterial resistance to antibiotics: modified target sites. Advanced drug delivery reviews, 57(10): 1471-1485.

[43] Langton, K.P., Henderson, P.J., Herbert, R.B., 2005. Antibiotic resistance: multidrug efflux proteins, a common transport mechanism?. Natural product reports, 22(4): 439-451.

[44] Laxminarayan, R., Duse, A., Wattal, C., Zaidi, A.K., Wertheim, H.F., Sumpradit, N., Vlieghe, E., Hara, G.L., Gould, I.M., Goossens, H., Greko, C., 2013. Antibiotic resistance- the need for global solutions. The Lancet infectious diseases, 13(12): 1057-1098.

[45] Leach, K.L., Swaney, S.M., Colca, J.R., McDonald, W.G., Blinn, J.R., Thomasco, L.M., Gadwood, R.C., Shinabarger, D., Xiong, L., Mankin, A.S., 2007. The site of action of oxazolidinone antibiotics in living bacteria and in human mitochondria. Molecular cell, 26(3): 393-402.

[46] Levy, S.B. and Marshall, B., 2004. Antibacterial resistance worldwide: causes, challenges and responses. Nature medicine, 10(12): 122.

[47] Levy, S.B., 2002. Factors impacting on the problem of antibiotic resistance. Journal of Antimicrobial Chemotherapy, 49(1): 25-30.

[48] Loeffler, J. and Stevens, D.A., 2003. Antifungal drug resistance. Clinical infectious diseases, 36(1):31-41.

[49] Martinez, J.L. and Baquero, F., 2000. Mutation frequencies and antibiotic resistance. Antimicrobial agents and chemotherapy, 44(7): 771-777.

[50] McManus, M.C., 1997. Mechanisms of bacterial resistance to antimicrobial agents. American Journal of Health-System Pharmacy, 54(12): 1420-1433.

[51] Misra, R., Morrison, K.D., Cho, H.J., Khuu, T., 2015. Importance of real-time assays to distinguish multidrug efflux pump inhibiting and outer membrane destabilizing activities in Escherichia coli. Journal of bacteriology.

[52] Nagai, K., Davies, T.A., Jacobs, M.R., Appelbaum, P.C., 2002. Effects of amino acid alterations in penicillin-binding proteins (PBPs) $1 \mathrm{a}, 2 \mathrm{~b}$, and $2 \mathrm{x}$ on PBP affinities of penicillin, ampicillin, amoxicillin, cefditoren, cefuroxime, cefprozil, and cefaclor in 18 clinical isolates of penicillinsusceptible, -intermediate, and-resistant pneumococci. Antimicrobial agents and chemotherapy, 46(5): 1273-1280. 
[53] Nagarajan, R., 1991. Antibacterial activities and modes of action of vancomycin and related glycopeptides. Antimicrobial agents and chemotherapy, 35(4): 605.

[54] Nikaido, H., 1998. Multiple antibiotic resistance and efflux. Current opinion in microbiology, 1(5): 516-523.

[55] Okeke, I.N., Laxminarayan, R., Bhutta, Z.A., Duse, A.G., Jenkins, P., O'Brien, T.F., PablosMendez, A., Klugman, K.P., 2005. Antimicrobial resistance in developing countries. Part I: recent trends and current status. The Lancet infectious diseases, 5(8): 481-493.

[56] Paphitou, N.I., 2013. Antimicrobial resistance: action to combat the rising microbial challenges. International journal of antimicrobial agents, 42: 25-28.

[57] Raghunath, D., 2008. Emerging antibiotic resistance in bacteria with special reference to India. Journal of biosciences, 33(4): 593-603.

[58] Reinert, R.R., Wild, A., Appelbaum, P., Lütticken, R., Cil, M.Y., Al-Lahham, A., 2003. Ribosomal mutations conferring resistance to macrolides in Streptococcus pneumoniae clinical strains isolated in Germany. Antimicrobial agents and chemotherapy, 47(7): 2319-2322.

[59] Roberts, M.C., Sutcliffe, J., Courvalin, P., Jensen, L.B., Rood, J., Seppala, H., 1999. Nomenclature for macrolide and macrolide-lincosamide-streptogramin B resistance determinants. Antimicrobial agents and chemotherapy, 43(12): 2823-2830.

[60] Spicknall, I.H., Foxman, B., Marrs, C.F., Eisenberg, J.N., 2013. A modeling framework for the evolution and spread of antibiotic resistance: literature review and model categorization. American journal of epidemiology, 178(4): 508-520.

[61] Strohl, W.R., 2004. Antimicrobials. In Microbial diversity and bioprospecting. American Society of Microbiology. pp. 336-355.

[62] Tanwar, J., Das, S., Fatima, Z., Hameed, S., 2014. Multidrug resistance: an emerging crisis. Interdisciplinary perspectives on infectious diseases, 2014.

[63] Tenover, F.C., 2006. Mechanisms of antimicrobial resistance in bacteria. American journal of infection control, 34(5):3-10.

[64] Vannuffel, P. and Cocito, C., 1996. Mechanism of action of streptogramins and macrolides. Drugs, 51(1): 20-30.

[65] Vannuffel, P., Di Giambattista, M., Morgan, E.A., Cocito, C., 1992. Identification of a single base change in ribosomal RNA leading to erythromycin resistance. Journal of Biological Chemistry, 267(12): 8377-8382.

[66] Wang, G.E. and Taylor, D.E., 1998. Site-Specific Mutations in the 23S rRNA Gene of Helicobacter pylori Confer Two Types of Resistance to Macrolide-Lincosamide-Streptogramin B Antibiotics. Antimicrobial agents and chemotherapy, 42(8): 1952-1958.

[67] Weisblum, B., 1995. Erythromycin resistance by ribosome modification. Antimicrobial agents and chemotherapy, 39(3): 577.

[68] Wilke, M.S., Lovering, A.L., Strynadka, N.C., 2005. $\beta$-Lactam antibiotic resistance: a current structural perspective. Current opinion in microbiology, 8(5): 525-533.

[69] Williams, D.H., Maguire, A.J., Tsuzuki, W., West well, M.S., 1998. An analysis of the origins of a cooperative binding energy of dimerization. Science, 280(5364): 711-714.

[70] Wright, G.D., 2005. Bacterial resistance to antibiotics: enzymatic degradation and modification. Advanced drug delivery reviews, 57(10): 1451-1470.

[71] Yoneyama, H. and Katsumata, R., 2006. Antibiotic resistance in bacteria and its future for novel antibiotic development. Bioscience, biotechnology, and biochemistry, 70(5): 1060-1075.

\footnotetext{
*Corresponding author.

E-mail address: Gudatachala9@gmail.com
} 